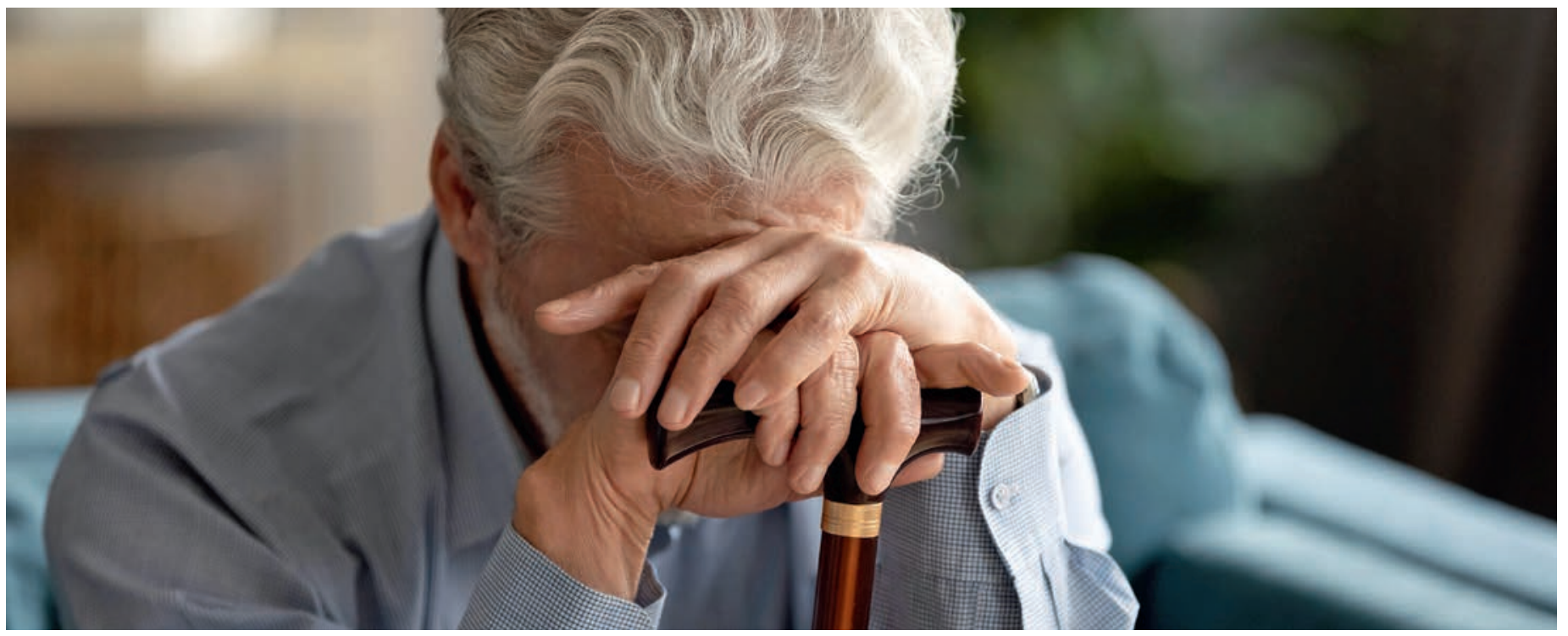

\title{
Den Altersfreitod nicht totschweigen
}

\section{Jürg Wilera, Marion Schafroth ${ }^{b}$}

${ }^{a}$ Vizepräsident EXIT Deutsche Schweiz; ${ }^{b}$ Dr. med., Präsidentin

Wenn hochbetagte und leidende Menschen sich für eine Freitodbegleitung entscheiden, obwohl sie nicht an einer tödlichen Krankheit leiden, ist Verständnis vonnöten innerhalb unserer Gesellschaft und im Besonderen innerhalb der Ärzteschaft.

Noch nie haben so viele alte Menschen in der Schweiz gelebt wie heute. Dabei wächst die Bevölkerungsgruppe der Hochaltrigen am schnellsten. So ist heute jeder zwanzigste Mensch 80-jährig und älter, in 25 Jahren dürfte es jeder zehnte sein. Im Gleichschritt mit der steigenden Anzahl pochen immer mehr von ihnen auf ihre Selbstbestimmung. Dies, weil sie ein Leben lang gelernt haben, die Weichen eigenständig zu stellen, zum Beispiel bei der Ausbildung, der Partnerwahl oder der Kinderfrage. Auch die steten Fortschritte der Medizin hinterlassen bei hochbetagten Menschen ihre Spuren: Immer mehr geraten in die Situation, dass ihr Leben durch medizinische Therapien und unterstützende Massnahmen auch dann aufrechterhalten werden kann, wenn sie dies nach einem erfüllten Leben gar nicht mehr wünschen und ihnen jegliche positive Zukunftsperspektive fehlt.

\section{Altersfreitod als Tabu}

Viele alte Menschen fragen sich zu Recht, weshalb sie am Lebensende - sollten sie ihre Gebrechen und ihr Leiden nicht mehr aushalten - diese wohl wichtigste Entscheidung nicht selbst oder zusammen mit Ange- hörigen fällen können. Ein Grund dafür ist, dass die Suizidhilfe für Hochbetagte ohne schwerste tödliche Krankheit in unserer Gesellschaft immer noch tabuisiert oder gar totgeschwiegen wird. Aufgrund dieser stillen moralischen Verurteilung gehen zu viele alte Menschen den Weg des gewaltsamen Suizids, der für alle Betroffenen schrecklich ist. Oder sie verbringen ihre letzten Lebensjahre in einem unglücklichen Leidenszustand, bis sie der natürliche Tod endlich vom nur noch als Last empfundenen Leben erlöst.

Weit verbreitet ist hierzulande die Meinung: Nur wer an einer tödlichen Krankheit leidet, darf Suizidhilfe in Anspruch nehmen. Diese Ansicht ist falsch. Tatsache vielmehr ist, dass sowohl der Europäische Gerichtshof für Menschenrechte als auch das Schweizerische Bundesgericht jedem Menschen das Grundrecht zubilligt, über die Art und den Zeitpunkt des eigenen Lebensendes zu entscheiden - sofern er urteilsfähig ist, also die Folgen seiner Entscheidungen abschätzen kann. Mit anderen Worten akzeptiert die Rechtsprechung, dass jeder urteilsfähige Mensch dieses Grundrecht der Entscheidungsfreiheit hat, losgelöst vom Alter, von allfälligen Krankheiten und Funktionseinschränkungen. Das Schweizer Strafgesetz schränkt nur ein, dass Suizidhilfe 
nicht aus "selbstsüchtigen Beweggründen» erfolgen darf, um zum Beispiel zu einer Erbschaft zu kommen.

\section{Ganz normale Option am Lebensende}

Weder das Heilmittelgesetz noch das Betäubungsmittelgesetz enthält Regelungen, welche die Suizidhilfe betreffen. Einzige Einschränkung hier: Das verwendete Sterbemedikament Natrium-Pentobarbital ist rezeptpflichtig, womit es zwingend ein Arzt verschreiben muss. Ärztliche Standesregeln und die Richtlinien der Schweizerischen Akademie der Medizinischen Wissenschaften (SAMW) sind nicht verbindliches Recht, sondern Handlungsempfehlungen Kurzum: Die Suizidhilfe ist in unserem Land rechtlich erlaubt, wenn ein Sterbewilliger urteilsfähig ist, die sogenannte Tatherrschaft innehat - also das Sterbemedikament selbst trinkt oder den Infusionshahn dazu selbst öffnet - sowie keine selbstsüchtigen Motive vorliegen. Nebst diesen rechtlichen Bestimmungen spricht aus ethischer Sicht nichts gegen einen selbstgewollten Freitod - auch im Alter: Er kann als ganz normale Option am Ende des Lebensweges angesehen werden.

Dennoch begleitet die Sterbehilfeorganisation EXIT keine kerngesunden betagten Menschen in den Freitod. Sie definiert den sogenannten Altersfreitod als besondere Kategorie der Freitodbegleitung. Der Non-ProfitVerein versteht darunter die Suizidhilfe für einen betagten Menschen, der nicht an einer tödlichen Krankheit leidet, aber wegen der Summe seiner Beschwerden und Leiden seine Lebensqualität als beeinträchtigt empfindet. Das subjektiv unerträgliche "Leiden im und am Alter» stellt das entscheidende Kriterium dar. Der Begriff «Leiden» umfasst die Verminderung von körperlichen Funktionen, abnehmende Sinnesleistungen und starke Defizite in der Leistungsfähigkeit.

\section{Das Wichtigste in Kürze}

- Die Suizidhilfe für Hochbetagte ohne schwerste tödliche Krankheit wird in der Schweiz noch immer tabuisiert.

- Aufgrund dieser stillen moralischen Verurteilung gehen zu viele alte Menschen den Weg des gewaltsamen Suizids, der für alle Betroffenen schrecklich ist, oder sie verbringen ihre letzten Lebensjahre in einem unglücklichen Leidenszustand.

- Die Suizidhilfe ist in der Schweiz rechtlich erlaubt, wenn ein Sterbewilliger urteilsfähig ist, die sogenannte Tatherrschaft innehat sowie wenn keine selbstsüchtigen Motive vorliegen - dieses Grundrecht auf Entscheidungsfreiheit ist also unabhängig vom Alter.

- Dennoch begleitet EXIT keine kerngesunden betagten Menschen in den Freitod: Das subjektiv unerträgliche «Leiden im und am Alter" stellt das entscheidende Kriterium dar.

\section{Begleitung von Geburt bis Tod}

Leid verursachen können neben Krankheiten zum Beispiel auch eine Schwerhörigkeit, eine Sehverminderung oder medizinisch nicht erfassbare Schwindelanfälle. Zudem werden bei der Beurteilung psychosoziale Faktoren und das Wissen um zu erwartendes Leiden berücksichtigt. Bereits seit Jahren fällt rund ein Drittel der Freitodbegleitungen von EXIT in die Kategorie des Altersfreitods und wird in der internen Statistik unter der Bezeichnung «Polymorbidität» erfasst.

Nun braucht es Ärzte, die ihre Patientinnen und Patienten von der Geburt bis zum Tod begleiten. Betagte Menschen wünschen am Lebensende, mit ihrer Ärztin oder ihrem Arzt über alle Aspekte offen sprechen zu können - dazu gehört manchmal die Alternative Freitodhilfe - und Unterstützung auf dem eingeschlagenen Weg zu finden. Aufgrund der Gesetzeslage sind Ärzte die Gatekeeper zum Sterbemedikament. Dennoch ist und bleibt Suizidhilfe eine freiwillige ärztliche Tätigkeit. Es gibt Ärzte, die nicht bereit sind, ein Rezept für Natrium-Pentobarbital auszustellen. In solchen Fällen vermittelt EXIT Kontakt zu einem Arzt, der Suizidhilfe mit seiner ethischen Haltung und somit seinem beruflichen Verständnis vereinbaren kann.

$\mathrm{Zu}$ hoffen ist, dass sich mit dem Wachstum der betagten Bevölkerung auch die zum Teil ablehnende Haltung der Ärzteschaft hinsichtlich Altersfreitod ändert. Damit betagte, leidende Menschen nicht unerwünscht lange leben müssen, sondern bei Bedarf mit der Unterstützung ihres vertrauten Hausarztes ihr Leben aufgrund von Leiden und fehlender Perspektive auf humane und würdevolle Weise beenden dürfen.

\section{Bildnachweis}

(C) Fizkes | Dreamstime.com (Symbolbild)

\section{L'essentiel en bref}

- En Suisse, l'assistance au suicide reste taboue pour les personnes très âgées qui ne sont pas atteintes d'une maladie lourde et mortelle.

- En raison de cette condamnation morale tacite, trop de personnes âgées choisissent le suicide violent, ce qui est terrible pour toutes les personnes concernées, ou finissent leurs jours dans un état de souffrance et de malheur.

- L'assistance au suicide est légale en Suisse si la personne concernée est capable de discernement, si elle a ce qu'on appelle la maîtrise de l'acte et en l'absences de motifs égoïstes - ce droit fondamental à la liberté de choix est donc indépendant de l'âge.

- Néanmoins, EXIT n'accompagne pas le suicide des personnes âgées foncièrement en bonne santé: le critère décisif est la "souffrance subjectivement insupportable durant la vieillesse et liée à celle-ci». 